\title{
Towards a behavioral theory of bias in signal detection
}

\author{
DIANNE McCARTHY and MICHAEL DAVISON \\ University of Auckland, Auckland, New Zealand
}

\begin{abstract}
A behavioral model for performance on signal-detection tasks is presented. It is based on a relation between response and reinforcement ratios which has been derived from both animal and human research on the distribution of behavior between concurrently available schedules of reinforcement. This model establishes the ratio of obtained reinforcements for the choice responses, and not the probability of stimulus presentation, as the effective biaser in signal-detection research. Furthermore, experimental procedures which do not control the obtained reinforcement ratio are shown to give rise to unstable bias contours. Isobias contours, on the other hand, arise only from controlled reinforcement-ratio procedures.
\end{abstract}

The theory of signal detection (Peterson, Birdsall, \& Fox, 1954; Tanner \& Swets, 1954; van Meter \& Middleton, 1954) holds the promise of extracting two independent measures to describe behavior in a detection task. The two measures are stimulus discriminability, a measure of the subject's ability to tell two stimulus conditions apart, and bias (or criterion), a measure of how performance can be changed by nonsensory motivational or payoff variables. Most research in contemporary psychophysics has placed primary emphasis upon the sensory performance of human subjects, and attempts to relate stimulus parameters to the physical properties of the stimuli, independently of bias, are well documented. As a result, rather less effort has been expended in the search for a bias parameter which remains invariant with changes in discriminability (Dusoir, 1975; Luce, 1963).

The term "bias" (or "criterion") has frequently been used in both an explanatory and a descriptive sense-often with serious confusion (Treisman, 1976). In addition, as Dusoir (1975) pointed out, there is no generally accepted way of measuring bias and, hence, there is little agreement on the true shape of empirical isobias contours. Dusoir suggested the need for a measure of bias which was unaffected by changes in variables which, on a priori grounds, might be expected to change only discriminability (e.g., stimulus values). The measure must, however, be affected by operations which should manipulate

The research reported here was supported entirely by the New Zealand University Grants Committee, to which organization we continue to be most grateful. We thank the Associate Editor, Dr. A. Kristofferson, and an anonymous reviewer for helpful comments, and also Michael Corballis for constructive discussion. Requests for reprints may be sent to Dianne McCarthy, Department of Psychology, University of Auckland, Private Bag, Auckland; New Zealand. bias (e.g., stimulus-presentation probability and payoff). Dusoir, reviewing the then-current theories of bias (e.g., Broadbent, 1971; Hardy \& Legge, 1968; Healy \& Jones, 1973; Luce, 1963; Parks, 1966; Thomas \& Legge, 1970; Treisman, 1964), found no measure of bias satisfying the above requirements.

Here, we review a behavioral approach to bias which: (1) unlike signal-detection theory, does not depend upon any a priori distribution assumptions; (2) relates the change in behavior to a change in a measurable independent variable; and (3) is based on a well-documented empirical relation in concurrent schedule choice behavior-the generalized matching law (Baum, 1974). Our interpretation results in a bias expression similar to that proposed by Luce (1963), who obtained a bias parameter, $b$, and a discriminability parameter, $\eta$, by applying choice theory (Luce, 1959) to the standard signal-detection paradigm. Like Luce, we also obtain a discriminability-free index of bias without relying upon any assumed underlying theoretical distributions. Furthermore, our model separates this bias measure into a constant and a variable component.

The paper opens by tracing the development of the generalized matching law (Baum, 1974) in the experimental analysis of choice behavior, and follows this with the presentation of a model for signal-detection performance based on the application of this law to the standard detection-theory payoff matrix (Davison \& Tustin, 1978). The remainder of the paper is devoted to a discussion of the implications of this model for the measurement of bias and the generation of isobias contours in animal psychophysics. In addition, we will show how the bias problem, as specified by Dusoir (1975), can be seen as a problem in defining biasing variables in relation to experimental procedures. We close with a brief discussion concerning the extent to which both animal and human psychophysics can be described by a behavioral theory. 


\section{Generalized Matching Law}

The generalized matching law (Baum, 1974) is a quantitative description of how stable performance in concurrent (CONC) and multiple (MULT) schedules is affected by changing the reinforcements, or payoffs, for the two (or more) performances. In concurrent schedules, two responses are available simultaneously and each is reinforced on a defined schedule. For example, in a concurrent variable-interval variable-interval (CONC VI VI) schedule, each of the two responses to two manipulanda is reinforced aperiodically. A typical manipulation would be to vary the mean intervals of the two VI schedules, so that different numbers of reinforcements would be obtained for the two responses. Other common manipulations would be to vary the magnitudes, delays, and types of reinforcements (see de Villiers, 1977). A multiple schedule is similar, except that the schedules are successively presented to the subject for a period of time with a distinctive stimulus associated with the operation of each schedule.

Studies of control by schedules of reinforcement have attempted to specify quantitatively how stablestate responding in the components of concurrent and multiple schedules may be controlled by the reinforcements obtained in each component schedule. The method of assessing the sensitivity of behavior to changes in reinforcement is to plot the ratio of the number of responses emitted on each alternative as a function of the ratio of the number of reinforcements obtained on each alternative (Baum, 1974; Baum \& Rachlin, 1969; Staddon, 1968).

If least squares linear regression lines are fitted between the logarithm of the response ratio and the logarithm of the obtained reinforcement ratio (Figure 1), the slope of the fitted line measures the sensitivity with which response allocation changes with re-

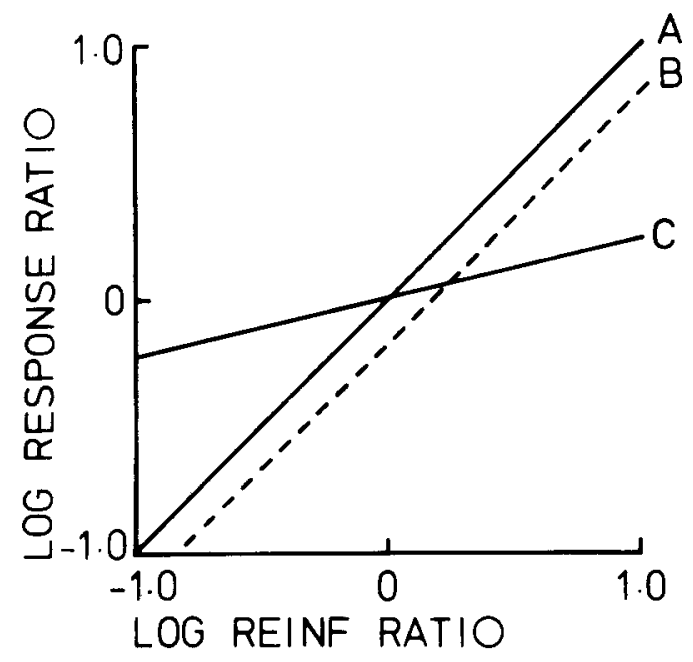

Figure 1. The logarithm of response ratios as a function of the logarithm of reinforcement ratios. Line $A$ represents strict matching. Line $B$ represents biased matching performance. Line $C$ represents undermatching. inforcement allocation (Baum, 1974; Lander \& Irwin, 1968). Stable performance generally conforms to the generalized matching law equation (Baum, 1974):

$$
\frac{P_{A}}{P_{B}}=c\left(\frac{R_{A}}{R_{B}}\right)^{a},
$$

where $P_{A}$ and $P_{B}$ are the number of responses emitted in the two components, and $\mathbf{R}_{A}$ and $\mathbf{R}_{B}$ are the number of reinforcements obtained from the component schedules. The exponent a reflects the sensitivity of the response ratio to changes in the ratio of obtained reinforcements (Lander \& Irwin, 1968). The value of $c$ describes inherent bias (Baum, 1974; McCarthy \& Davison, 1979), a constant preference over all experimental conditions unaffected by changes in the obtained reinforcement distribution between the two alternatives.

The values of $a$ and $c$ are obtained from the slope and intercept, respectively, of a least squares line fitted to the logarithmic data, that is:

$$
\log \left(\frac{P_{A}}{P_{B}}\right)=a \log \left(\frac{R_{A}}{R_{B}}\right)+\log c .
$$

If the ratios match $(a=c=1)$, as they would according to the strict matching law (Herrnstein, 1970), then a line of unit slope would pass through the point $(0,0)$; see Line $A$ in Figure 1 . That is, when reinforcements are distributed equally between the two alternatives (unit ratio), an equal number of responses will be emitted on each alternative.

Baum (1974) noted that two kinds of deviation from strict matching, in terms of a or c, are observed: the subject may over- or underestimate reinforcement differences between the alternatives for various reasons, yielding a value of a different from unity. This is called overmatching or undermatching, depending on its direction. An undermatching relation, for example, is shown as Line $C$ in Figure 1. Or the subject may be inclined to over- or underrespond to one alternative or the other, independent of reinforcement. This is inherent bias, and is represented by a nonunit value of $c$. Such a constant bias, given by the antilog of the intercept, shows up as a constant displacement from the matching diagonal, as indicated by Line B in Figure 1. Frequently, combinations of both undermatching and inherent bias are reported (Baum, 1974).

Undermatching. After training to a criterion of stability (typically 15 to $30 \mathrm{~h}$ ), the values of the exponent $\mathrm{a}$ in Equation 1 are between .80 and 1.0 for CONC VI VI schedule performance (Lobb \& Davison, 1975; Myers \& Myers, 1977), and about .33 for multiple schedule performance (Lander \& Irwin, 1968). Other schedule combinations yielding values for a of less than unity include concurrent fixed-interval variable-interval (CONC FI VI) (Lobb \& Davison, 1975; Nevin, 1971; Trevett, Davison, \& Williams, 
1972), MULT FI FI and MULT VI VI (Barron \& Davison, 1972; Lander \& Irwin, 1968), and CHAIN FI FI (Davison, 1974) schedules. Undermatching has also been found with concurrent differentialreinforcement-of-low-rate schedules (Staddon, 1968). Response ratio has been found to undermatch magnitude of reinforcement (Schneider, 1973; Todorov, 1973). Also, for CONC VI VI schedules when qualitatively different reinforcers are arranged for each component, response ratios have been found to undermatch reinforcement ratios (Hollard \& Davison, 1971; Mathews \& Temple, 1979).

Inherent bias. In the generalized matching law, an inherent bias ( $\log c$ in Equation 2) refers to a constant preference for one response over another independent of the reinforcement ratio (Baum, 1974). Such an inherent bias can arise out of the equipment (e.g., one response manipulandum more difficult to execute than another) or out of the subject (perhaps a preference for responding to one color rather than to another).

In general, inherent bias is therefore a nonunit ratio of a preference-controlling variable across the two responses, and this ratio remains constant when another variable is manipulated. A further example might be slightly different reinforcement durations (4 sec vs. $3 \mathrm{sec}$ of grain for pigeons, say) for the two responses. If this occurred in an experiment in which the numbers of reinforcements for the two responses were varied over experimental conditions, an inherent bias (excluding other sources of bias) of $.5 \times \log$ $(4 / 3)$, or .06 , would be expected (Schneider, 1973; Todorov, 1973). The multiplier, .5, reflects the general sensitivity of choice behavior to reinforcement duration changes.

In the generalized matching law, the term "bias" has generally been used only in its constant sense. When, however, a behavior-controlling variable is not kept constant (to provide a bias), but is systematically varied, it changes behavior with a certain sensitivity (a in Equation 2). The sensitivity parameter a measures the relation between changes in a biaser and changes in behavior. When one biaser is varied and another is kept constant, precise estimates of both the value of the constant biaser and the sensitivity with which behavior changes with the varied biaser can be obtained from a number of data points. While these could be estimated from only two data points, it is clearly preferable to use a least squares fit.

Exactly the same situation applies in signal-detection theory. Point estimates of discriminability and "bias" (or criterion) may be obtained from each condition comprising two choice proportions (hits and false alarms), but it is preferable to vary systematically one biaser to obtain more precise estimates of the other, constant, biaser. For example, the payoff matrix may be varied (i.e., criterion changed) to obtain an estimate of discriminability.

\section{Matching Model of Signal Detection}

By applying the generalized matching law (Equation 1) to the standard detection-theory payoff matrix, Davison and Tustin (1978) derived a measure of response bias, independent of discriminability and analogous to that of signal-detection theory. The following section traces the development of this approach.

In the standard yes-no detection task, the subject is trained to emit one response $\left(P_{1}\right)$ when one stimulus $\left(S_{1}\right)$ is presented and another response $\left(P_{2}\right)$ when a different stimulus $\left(\mathrm{S}_{2}\right)$ is presented. With two stimuli and two responses, four possible outcomes are defined. Figure 2 is a general stimulus-response matrix showing the events in a typical yes-no detection procedure. In this figure, $\mathrm{W}, \mathrm{X}, \mathrm{Y}$, and $\mathrm{Z}$ refer to the cells of the matrix. For example, with $P$ denoting responses and $R$ denoting reinforcers, $P_{w}$ is the number of responses in cell $W$ and $R_{Z}$ is the number of reinforcements obtained in cell $\mathrm{Z} . \mathrm{S}_{1}$ and $\mathrm{S}_{2}$ are two discriminative stimuli which may be related on the same physical dimension, or which may be related by one having an additive property to the other (e.g., noise, signal-plus-noise), or which may be unrelated.

$P_{1}$ and $P_{2}$ are the two choice responses which may be emitted (e.g., "yes" and "no," or a left-key response and a right-key response). Correct responses are $P_{1}$ in the presence of $S_{1}\left(P_{w}\right.$, hits) and $P_{2}$ in the presence of $S_{2}\left(P_{2}\right.$, correct rejections). Incorrect responses are $P_{2}$ in the presence of $S_{1}\left(P_{x}\right.$, misses) and $P_{1}$ in the presence of $S_{2}\left(P_{y}\right.$, false alarms $)$. In general, correct responses are reinforced with, perhaps, food or brain stimulation for animal subjects and money, points, or feedback for humans. Sometimes payoffs for correct responses are given each time a correct response is emitted (e.g., Hume, 1974a, 1974b; Hume

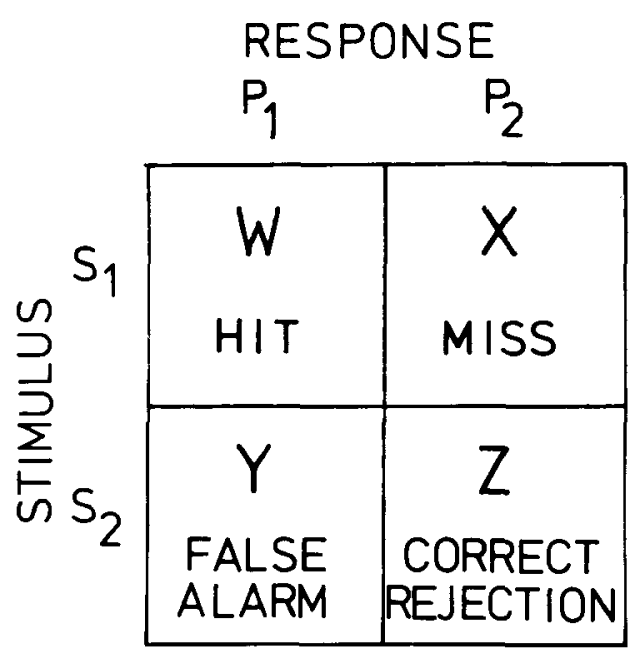

Figure 2. The matrix of stimulus and response events in a typical yes-no detection procedure. The cells of the matrix are denoted by $W, X, Y$, and $Z$. 
\& Irwin, 1974), and sometimes it is given intermittently on VI schedules (McCarthy \& Davison, 1979)' or on probabilistic variable-ratio schedules (e.g., Elsmore, 1972; McCarthy \& Davison, 1979, 1980a, 1980b; Stubbs, 1976). Usually, incorrect responses have no consequence or are punished in some way (e.g., time-out with animals; Hume, 1974b). The detection-theory payoff matrix, on the other hand, is a description of the arranged contingent events (reinforcers and punishers) for the various responses in the situation.

Davison and Tustin (1978) viewed the yes-no detection task as two concurrent reinforcement-extinction schedules, each operating under a distinctive stimulus. The generalized matching law suggests that if the two stimuli, $S_{1}$ and $S_{2}$, are indistinguishable, the distribution of total left and right responses would be determined by the distribution of reinforcers for left and right responses (cells $\mathrm{W}$ and $\mathrm{Z}$ in Figure 2). Davison and Tustin suggested that, as the stimuli become more discriminable, performance would move toward $P_{1}$ in $S_{1}$ and toward $P_{2}$ in $S_{2}$ (Figure 2), and that this movement could be described by a further additive quantity in a generalized matching law equation (Equation 2). Thus, Davison and Tustin proposed two generalized matching law equations to describe behavior in the presence of each of the two stimuli.

When only correct responses are reinforced (i.e., $\mathrm{R}_{\mathrm{x}}=\mathrm{R}_{\mathrm{y}}=0$ ), Davison and Tustin wrote:

Given $S_{1}$ :

$$
\log \left(\frac{P_{w}}{\mathbf{P}_{\mathrm{x}}}\right)=\mathrm{a}_{\mathrm{T}_{1}} \log \left(\frac{\mathbf{R}_{\mathrm{w}}}{\mathbf{R}_{\mathrm{z}}}\right)+\log \mathrm{d}+\log \mathrm{c},
$$

and given $\mathrm{S}_{\mathbf{2}}$ :

$$
\log \left(\frac{\mathrm{P}_{\mathrm{y}}}{\mathrm{P}_{\mathrm{z}}}\right)=\mathrm{a}_{\mathrm{r}_{\mathrm{z}}} \log \left(\frac{\mathbf{R}_{\mathrm{w}}}{\mathbf{R}_{\mathrm{z}}}\right)-\log \mathrm{d}+\log \mathrm{c},
$$

where $P$ and $R$ denote number of responses emitted and number of reinforcements obtained, respectively, and the subscripts refer to the cells of the matrix in Figure 2.

The parameters $a_{r_{1}}$ and $a_{r_{2}}$ are the sensitivities of behavior to changes in reinforcements, and $\log c$ is inherent bias. Log $d$ measures the discriminability of the two stimuli (Davison \& Tustin, 1978; McCarthy \& Davison, 1979). As stimulus separation increases, behavior moves toward $P_{1}$ in the presence of $S_{1}$, and away from $P_{1}$ in the presence of $S_{2}$. Since the numerators in both Equations 3 and 4 are the $P_{1}$ response category, $\log d$ is positive in Equation 3 and negative in Equation 4.

If we assume $a_{r_{1}}=a_{r_{2}}=a_{r}$, Equations 3 and 4 can also be used to specify how a measure of response bias may be obtained independently of discrimina- bility. Adding Equation 4 to Equation 3 to remove the effects of discriminability gives:

$$
\log \left(\frac{\mathbf{P}_{\mathrm{w}}}{\mathbf{P}_{\mathbf{x}}}\right)+\log \left(\frac{\mathbf{P}_{\mathbf{y}}}{\mathbf{P}_{\mathbf{z}}}\right)=2 \mathrm{a}_{\mathrm{r}} \log \left(\frac{\mathbf{R}_{\mathrm{w}}}{\mathbf{R}_{\mathbf{z}}}\right)+2 \log \mathrm{c} .
$$

This equation relates behavior in the presence of the two stimuli to the combined effects of inherent bias $(\log \mathrm{c})$, and the biasing effect of changing the reinforcement ratio in the two stimuli. Thus:

$$
\begin{aligned}
& a_{r} \log \left(\frac{R_{w}}{R_{z}}\right)+\log c \\
&=.5\left[\log \left(\frac{P_{w}}{P_{x}}\right)+\log \left(\frac{P_{y}}{P_{z}}\right)\right] .
\end{aligned}
$$

We call the measure on the right-hand side of Equation 6 response bias (McCarthy \& Davison, 1979), and it provides the same behavioral estimate of "bias" (or criterion) as that used in signal-detection theory. The left-hand side of Equation 6 specifies the environmental conditions which produce the response bias. Thus, a discriminability-free estimate of response bias is given by:

$$
\text { Response bias }=\left(\frac{\mathbf{P}_{w}}{\mathbf{P}_{x}} \cdot \frac{\mathbf{P}_{y}}{\mathbf{P}_{z}}\right)^{1 / 2} .
$$

This measure is equivalent (in terms of the matrix in Figure 2) to the reciprocal of that given by Luce (1963), namely:

$$
\mathbf{b}=\left[\frac{\operatorname{Pr}(\mathrm{N} / \mathrm{s})}{\operatorname{Pr}(\mathrm{Y} / \mathrm{s})} \cdot \frac{\operatorname{Pr}(\mathrm{N} / \mathrm{n})}{\operatorname{Pr}(\mathrm{Y} / \mathrm{n})}\right]^{1 / 2},
$$

where $\operatorname{Pr}(\mathrm{N} / \mathrm{s})$ is the probability of a miss, $\operatorname{Pr}(\mathrm{N} / \mathrm{n})$ is the probability of a correct rejection, $\operatorname{Pr}(\mathrm{Y} / \mathrm{s})$ is the probability of a hit, and $\operatorname{Pr}(\mathrm{Y} / \mathrm{n})$ is the probability of a false alarm.

Unlike Luce's (1963) measure, however, the present behavioral approach distinguishes clearly between two sources of response bias: (1) biases arising from different numbers of reinforcements for the two choice responses (or different magnitudes, etc.; McCarthy \& Davison, 1979), and (2) a constant bias $(\log \mathrm{c})$, which may arise from either the requirements of the experiment (e.g., response production vs. response omission; different forces required to operate response manipulanda) or from the subject itself. Such a constant bias is termed inherent bias. Both sources of response bias-reinforcement bias and inherent bias-are subsumed under the rubric of "criterion" in signal-detection research. Our approach partials response bias (or criterion) into a variable (i.e., reinforcement) component and a constant (i.e., inherent) component. 
Equations 3 and 4 can also be used to obtain a measure of stimulus discriminability uncontaminated by response bias. Again assuming $a_{r_{1}}=a_{r_{2}}$, Equation 4 can be subtracted from Equation 3 to eliminate the effects of reinforcement bias and inherent bias on detection performance. Thus:

$$
\log \left(\frac{\mathbf{P}_{\mathbf{w}}}{\mathbf{P}_{\mathbf{x}}}\right)-\log \left(\frac{\mathbf{P}_{\mathbf{y}}}{\mathbf{P}_{\mathbf{z}}}\right)=2 \log \mathrm{d} .
$$

An estimate of discriminability, independent of response bias, is, therefore, given by:

$$
\mathrm{d}=\left(\frac{\mathbf{P}_{\mathrm{w}}}{\mathbf{P}_{\mathbf{x}}} \cdot \frac{\mathbf{P}_{\mathbf{z}}}{\mathbf{P}_{\mathbf{y}}}\right)^{1 / 2}
$$

Davison and Tustin (1978) noted that discriminability, as measured by Equation 9, was identical to discriminability indices used by some signal-detection theorists (e.g., Luce, 1963) and equivalent to that used by others (e.g., Green \& Swets, 1966). Luce (1963), for example, measures discriminability as:

$$
\eta=\left[\frac{\operatorname{Pr}(\mathrm{N} / \mathrm{s})}{\operatorname{Pr}(\mathrm{Y} / \mathrm{s})} \cdot \frac{\operatorname{Pr}(\mathrm{Y} / \mathrm{n})}{\operatorname{Pr}(\mathrm{N} / \mathrm{n})}\right]^{1 / 2},
$$

which, in terms of the matrix in Figure 2, is the reciprocal of the expression given by Equation 9 of the Davison and Tustin model.

In essence, then, Davison and Tustin (1978) showed how independent measures of response bias and discriminability can be derived from an analysis of detection performance in terms of the matching of response ratios to reinforcement ratios. Perhaps the most salient features of their model, in relation to the present paper, include a partialing out of the sources of response bias, in particular, the influence of inherent bias and a clarification of the role of reinforcement bias in detection theory. ${ }^{1}$

\section{Implications of the Matching Model of Detection Performance for the Measurement of Response Bias in Animal Psychophysics}

In a series of experimental studies using pigeons as subjects, we have investigated the utility of this matching approach to signal-detection performance. Of particular concern for the present paper are our attempts to manipulate response bias by varying stimuluspresentation probability and obtained reinforcement ratios, and to uncover the role played by experimental procedures in the generation of isobias contours. In addition, we will show how the relation commonly found between obtained and optimal response biases in detection theory can be explained in terms of the Davison and Tustin (1978) model.

Role of stimulus-presentation probability as a biaser. Contemporary psychophysical research using both animal and human subjects has shown that, while a subject's ability to discriminate between two stimuli remains constant, he may be induced to change his response probabilities in the presence of each of the two stimuli. In these studies, response bias (or criterion) has commonly been manipulated by varying the probability of presenting one of the two stimuli (e.g., Clopton, 1972; Elsmore, 1972; Galanter \& Holman, 1967; Hume, 1974a, 1974b; Hume \& Irwin, 1974; Markowitz \& Swets, 1967; Schulman \& Greenberg, 1970; Swets, Tanner, \& Birdsall, 1961; Terman \& Terman, 1972). We have shown, however, that response bias is not a function of stimuluspresentation probability but, rather, is a function of the relative reinforcement frequency obtained for the choice responses.

In signal-detection research with animals, reinforcements for correct responses are often arranged in one of two ways: reinforcement for every correct response or reinforcement delivered probabilistically for correct responses. In both of these procedures, the number of reinforcements obtained for a given choice response covaries with stimulus-presentation probability (SPP). In other words, changing the probability of presenting the two stimuli changes the distribution of reinforcers between the response categories $P_{1}$ and $P_{2}$. For example, when SPP is $.9,9 / 10$ ths of the reinforcers will be obtained for correct responses emitted in the presence of one of the two stimuli. Thus, variations in SPP are confounded with variations in the obtained reinforcement ratio, and the reported biasing effect of SPP could be simply the result of the changing distribution of reinforcements.

We investigated this possibility by examining the performance of pigeons trained to detect differences between two light intensities under three experimental procedures (McCarthy \& Davison, 1979). All three procedures were standard signal-detection yesno analogues in which the center key of a three-key operant chamber was lit by either $S_{1}\left(33 \mathrm{~cd} / \mathrm{m}^{2}\right)$ or $\mathrm{S}_{2}\left(7 \mathrm{~cd} / \mathrm{m}^{2}\right)$ according to set probabilities. These probabilities ranged from .1 to .9 in steps of .2. Following presentation of either $S_{1}$ or $S_{2}$ on the center key, a peck on the center key turned on the two side keys which were illuminated red (left) and green (right). On $S_{1}$ trials, when the more intense stimulus was presented on the center key, a peck on the left key was defined as "correct." On $S_{2}$ trials, when the less intense stimulus was presented on the center key, a peck on the right key was "correct."

Correct responses produced either a 3-sec magazine light or, in addition, 3-sec access to wheat. Incorrect responses (that is, pecks on the left key after $S_{2}$ presentations and pecks on the right key after $S_{1}$ presentations) produced 3-sec blackout during which all chamber lights were extinguished, and responses were ineffective. The center-key light remained on 
until food, magazine light, or blackout had been produced, after which a new trial began. A noncorrection procedure was used throughout the experiment, the probability of occurrence of either $S_{1}$ or $S_{2}$ on the center key being independent of accuracy on the previous trial.

The difference between the three procedures was the way in which food reinforcement was arranged for correct responses. The first procedure was a standard signal-detection design in which the probability of occurrence of $S_{1}$ on the center key (SPP) was varied, and the number of reinforcements obtained $\left(R_{w}, R_{z}\right)$ for the two correct responses $\left(P_{w}, P_{z}\right)$ was allowed to covary with changes in the ratio of the frequencies of stimulus presentation. This procedure is an uncontrolled reinforcement-ratio procedure, and it is typical of most signal-detection research. In the second procedure, SPP was again varied, but equal numbers of reinforcements were obtained for the two correct responses. The third procedure held SPP constant at .7 , and the number of food reinforcements obtained for correct responses was varied. Procedures 2 and 3 are thus controlled reinforcement-ratio procedures, because the reinforcement ratio is set and cannot covary with either response ratios (preference) or SPP.

In this experiment, as in all animal psychophysical studies, the subjects were trained on each experimental condition until a strict criterion of stable performance was reached. Data were collected over five sessions of this stable performance. Training then commenced on the next experimental condition with changed reinforcement (or stimulus) parameters.

We found that behavior changed reliably in Procedure 1 , in which both the reinforcement ratio and SPP were varied, and in Procedure 3, in which the relative reinforcement ratio alone was varied. These behavioral changes were shown as positive slopes for Equations 3 and 4, and significant trends in the point estimates of response bias (Equation 7) as a function of the logarithm of the obtained reinforcement ratio. However, no significant trend occurred in the point estimates of response bias in Procedure 2 when SPP alone was varied.

As SPP manipulation produced no systematic change in the relative frequencies of the choice responses, we concluded that SPP was not a biaser per se, and that its apparent biasing effect arose from changes in the obtained reinforcement ratio. In other words, the variation of the obtained reinforcement ratio alone controlled or biased performance. Variations in SPP did, however, allow measures of stimulus discriminability (Equation 9) to be extracted from the data. As expected, discriminability remained constant across the three procedures.

Optimal bias. In signal-detection theory, optimal biases which maximize some aspects of payoff (such as overall reinforcement probabilities) are often spec- ified. Typically, in the normative version of detection theory, the critical value of the likelihood ratio, designated $\beta$, is chosen to maximize expected payoff (Green \& Swets, 1966). The expected value of a decision outcome represents the value or cost associated with that outcome, weighted by the probability of that outcome's occurring. Thus, if a subject chooses a decision rule which maximizes expected payoff, his optimal likelihood-ratio criterion, $\beta_{\text {opt }}$, is represented by the expression:

$$
\beta_{\text {opt }}=\frac{\operatorname{Pr}\left(S_{2}\right)}{\operatorname{Pr}\left(S_{1}\right)} \cdot \frac{V_{z}-V_{y}}{V_{w}-V_{x}},
$$

where $\operatorname{Pr}\left(S_{2}\right)$ and $\operatorname{Pr}\left(S_{1}\right)$ represent the a priori probabilities of stimulus presentation, $V_{z}$ and $V_{w}$ are the values associated with correct responses $\left(\mathrm{P}_{\mathrm{z}}\right.$ and $\left.\mathrm{P}_{\mathrm{w}}\right)$, and $V_{y}$ and $V_{x}$ are the costs associated with incorrect responses $\left(P_{y}\right.$ and $\left.P_{x}\right)$. The subscripts refer to the cells of the matrix in Figure 2.

Such values and costs are, however, poorly defined by signal-detection theorists (e.g., Egan, 1975). Hume (1974a, 1974b) specified value and cost as the number of brain stimulations for each correct response and the duration of blackout for each incorrect response, respectively. The obtained reinforcement ratio, on the other hand, is a variable which has not been considered by detection theorists. The detection-theory payoff matrix, denoting arranged payoff, specifies the values and costs of the payoffs. It does not specify the relative frequency at which the payoffs occur (i.e., obtained payoff). Obtained, rather than arranged, reinforcement ratio is the critical variable both in research on matching and in the present behavioral model of signal detection. As we have suggested, it is the ratio of obtained reinforcers, as well as their momentary value (e.g., quality, magnitude, or immediacy), which determines reinforcement bias.

In terms of the results reported above (McCarthy \& Davison, 1979), Equation 10 implies that, as the value of the payoff remained constant in all three procedures, response bias should simply be a function of $\operatorname{Pr}\left(\mathrm{S}_{1}\right) / \operatorname{Pr}\left(\mathrm{S}_{2}\right)$. This implies that response bias should have changed in Procedures 1 and 2, but not in Procedure 3. Clearly, then, Equation 10 is not consistent with our results, which showed responsebias changes in Procedures 1 and 3, but not in Procedure 2 .

It remains, therefore, to consider response bias as in the Davison and Tustin (1978) model, that is:

$$
\text { Response bias }=a_{r} \log \left(\frac{R_{w}}{R_{z}}\right)+\log c \text {. }
$$

Here, $a_{r}$ is a free parameter which may take any value, but which would be expected to fall between .5 and 1.0 (Baum, 1979) on the basis of extensive research 
on schedule control. If a standard yes-no detection procedure with reinforcement for each correct response is used, the reinforcement ratio $\left(R_{w} / R_{z}\right)$ will approximate the ratio $\operatorname{Pr}\left(\mathrm{S}_{1}\right) / \operatorname{Pr}\left(\mathrm{S}_{2}\right)$, and a strategy such as maximizing expected value will give an $a_{r}$ value of 1.0. A value of $a_{r}$ of less than 1.0 would represent a failure to maximize expected utility and, perhaps, the use of a different strategy. While, in a sense, the $a_{r}$ parameter is weak, it does allow changes in response bias in signal-detection experiments to be related directly to another extensive area of researchschedule control. It is worthwhile noting that, in this latter area, no particular strategy, such as maximizing expected value, has ever been demonstrated (e.g., Herrnstein \& Heyman, 1979).

In human psychophysical experiments, subjects generally fail to behave exactly in the way specified by Equation 10. Typically, when $\beta_{\text {opt }}$ is relatively large, the bias exhibited by the subject $\left(\beta_{\text {obt }}\right)$ is not as high as the optimal bias, and when $\beta_{\text {opt }}$ is relatively small, $\beta_{\text {obt }}$ is not as low as the optimal bias. Such conservative behavior has been attributed to the subject's awareness in psychophysical settings that the experimenter's principal interest is in a sensory process so that he believes that all "yes" or all "no" responses are either not possible or not desired (Green \& Swets, 1966).

Rather than defining behavior as suboptimal, however, it is more profitable to define the expected degree of bias by Equation 11 above. This expression does not specify strict matching between expected and obtained bias, but, rather, allows the possibility of undermatching $\left(a_{r}<1.0\right)$ for a more general statement of the relation between response and reinforcement ratios. Certainly, undermatching is the norm with various biasers (e.g., reinforcement magnitude; Schneider, 1973, and Todorov, 1973), and it may also be the norm with various schedule combinations (e.g., CONC FI VI; Lobb \& Davison, 1975, and Trevett, Davison, \& Williams, 1972).

Any general description of detection performance must account for different biasers produced by different response and schedule combinations, and must allow for the possibility of undermatching. Figure 3 shows a typical plot of obtained bias as a function of optimal bias (Hume \& Irwin, 1974). Optimal bias is here defined as the ratio of the probability of one stimulus's occurring to the probability of the other stimulus's occurring on any trial, which, in terms of the present model, assuming an uncontrolledreinforcement ratio procedure, is correlated with the obtained reinforcement ratio, $\mathbf{R}_{\mathbf{w}} / \mathbf{R}_{\mathbf{z}}$.

Figure 3 shows clearly that the obtained bias $\left(\beta_{\text {obt }}\right)$ undermatches optimal bias $\left(\beta_{\text {opt }}\right)$, as would be expected from schedule-control research. Extracting the data from Figure 3, the slopes and intercepts of the relation are shown in Table 1 . As Table 1 shows,

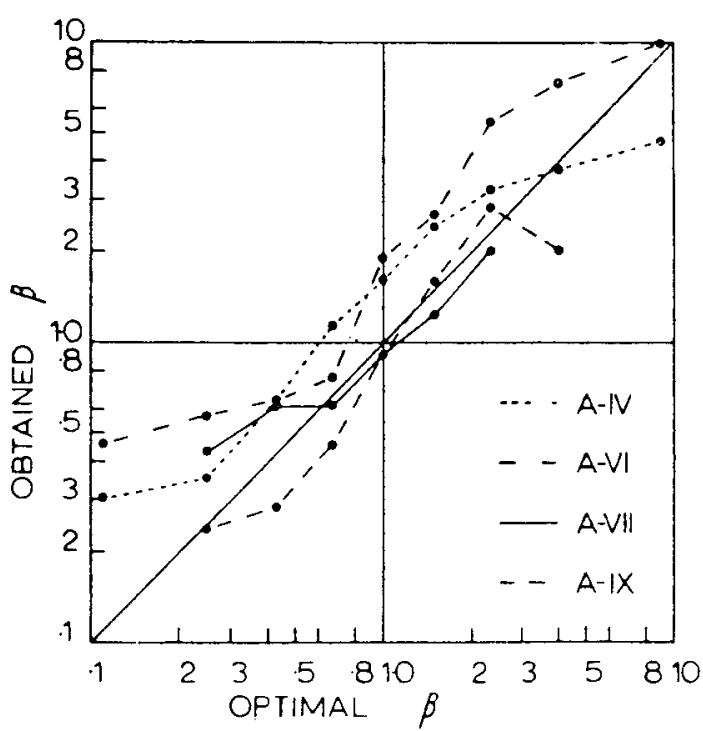

Figure 3. Data reported by Hume and Irwin (1974). The relation between obtained response criteria $\left(\beta_{\text {obt }}\right.$ ) and optimal response criteria $\left(\beta_{\text {opt }}\right)$. The coordinates are logarithmically spaced, and the major diagonal represents perfect correspondence between obtained and optimal criteria. (Copyright 1971 by the Society for the Experimental Analysis of Behavior, Inc.)

there is an undermatching relation (slope less than unity) between $\beta_{\text {opt }}$ and $\beta_{\text {obt }}$, and there are large inherent biases (e.g., $\log \mathrm{c}$ of .27 for Rat AVI). Nevertheless, these results can be described by Equation 11 of the Davison-Tustin model.

Independence of response bias from discriminability. As noted by Dusoir (1975), current measures of "bias" (or criterion) in signal-detection theory cannot be unequivocally shown to be independent of discriminability. The present model assumes that the behavioral effects of response bias and discriminability are additive in logarithmic terms (Equations 3 and 4), and hence, there is no interaction between these two independent variables. There can be, however, many alternative models which do not treat response bias and discriminability as simply additive in their effects on behavior. Rather, they include interactions between these two variables.

In signal-detection theory, for instance, it has long been accepted that uncontrolled cognitive factors (e.g., criterion variance) can contaminate estimates

Table 1

Slopes and Intercepts for the Relation Between Obtained Response Criteria and Optimal Response Criteria as Plotted in Figure 3

\begin{tabular}{ccc}
\hline Subject & Slope & Intercept \\
\hline AVI & .84 & .27 \\
AIV & .72 & .14 \\
AVII & .67 & -.01 \\
AIX & .96 & -.09 \\
\hline
\end{tabular}


of discriminability. The detection-theory concept of criterion, or response bias, is formulated in such a way as to make measures of discriminability invariant with changes in criterion. The location of the criterion depends upon the value and cost of making correct and incorrect responses and on the probability of stimulus presentation (Equation 10). While the criterion is assumed to be stable in signal-detection tasks, if it is not, it becomes an additional source of variance in the statistical model (Tanner \& Swets, 1954; Treisman, 1977; Wickelgren, 1968). In animal psychophysical studies, for example, there are numerous reports of uncontrolled bias shifts (e.g., Irwin \& Terman, 1970; Terman \& Terman, 1972).

We investigated empirically the independence of response bias and discriminability in the present model by examining the biasing effect of the reinforcement ratio on detection performance at two different levels of discriminability (McCarthy \& Davison, $1980 \mathrm{~b})$. Here, the reinforcement ratio was varied for both an easy and a difficult discrimination to see whether similar reinforcement sensitivities were obtained at both discriminability levels.

Again, using a standard signal-detection yes-no analogue, six pigeons were trained to detect differences between two white stimuli, $S_{1}$ and $S_{2}$, differing in duration and arranged probabilistically on the center key of a three-key operant chamber. For the easy discrimination, $S_{1}$ was $5 \mathrm{sec}$ and $S_{2}$ was $30 \mathrm{sec}$. For the difficult discrimination, $S_{1}$ was $20 \mathrm{sec}$ and $S_{2}$ was $30 \mathrm{sec}$. The probability of occurrence of $S_{1}$ on the center key (SPP) was varied from .1 to .9 in steps of .2 , for both pairs of stimuli. The obtained reinforcement ratio for correct responses emitted on the two side keys (left after $S_{1}$, right after $S_{2}$ ) was uncontrolled, allowing it to covary with changes in stimuluspresentation probability.

Discriminability, as measured by Equation 9, was indeed different for the two discriminations. For the 5-sec vs, 30-sec conditions, the mean value of discriminability (log d), averaged across the six birds, was 1.48 , a significantly higher value than that obtained for the 20-sec vs. 30 -sec conditions (mean = .39). In addition, there was no systematic change in discriminability as a function of the obtained reinforcement ratio for either the easy or the difficult discrimination.

Point estimates of response bias (Equation 7), on the other hand, significantly increased as a function of the obtained reinforcement ratio for both discriminability levels (Figure 4a). While bias estimates were similar for the two discriminability levels, the range over which response bias varied was larger for the difficult discrimination than for the easy discrimination (Figure 4a). This result is attributable to the use of an uncontrolled reinforcement-ratio procedure, with resulting deviations of the obtained reinforce-

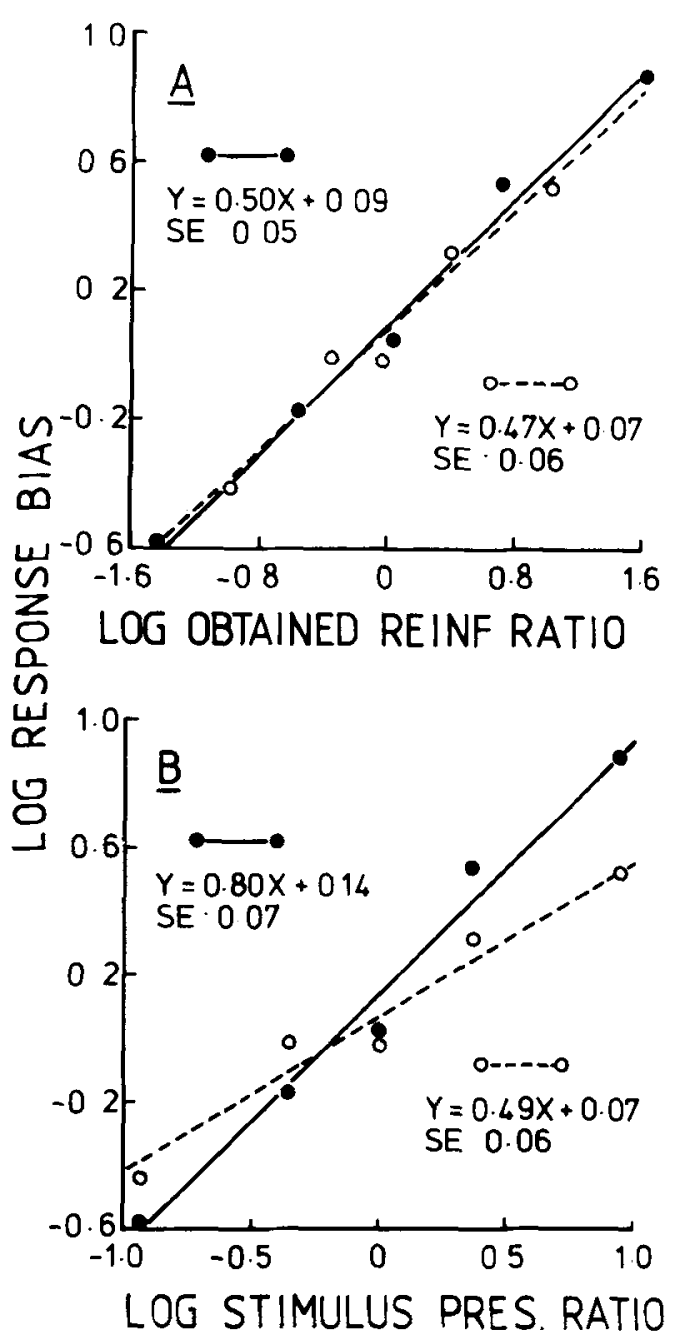

Figure 4. Point estimates of response bias (Equation 7) for the 5-sec vs. 30-sec conditions (unfilled circles) and the 20-sec vs. 30 -sec conditions (filled circles). A shows these point estimates as a function of the logarithm of the obtained reinforcement ratio, and $B$ as a function of the logarithm of the stimulus-presentation ratio. The best-fitting straight line by the method of least squares and the standard error (SE) of the estimate are shown for each stimulus condition.

ment ratio from the arranged reinforcement ratio for the difficult discrimination. This deviation can be seen by plotting the point estimates of response bias as a function of the logarithm of the stimuluspresentation ratio, as in Figure $4 \mathrm{~b}$. The slope for the difficult discrimination here is greater than that for the easy discrimination (slopes $=.80$ and .49 , respectively). However, when both sets of data were plotted as a function of the obtained reinforcement ratio (Figure 4a), no differences in the slopes were found (slope $=.47$ for the easy discrimination; slope $=.50$ for the difficult discrimination). This result, then, again underlines the importance of recognizing the obtained reinforcement ratio, and not the probability 
of stimulus presentation, as the effective biaser. In addition, it provides support (as have most of our experiments) for the assumption of equal reinforcement sensitivities (i.e., $a_{r_{1}}=a_{r_{2}}$ ) in Equations 3 and 4 . However, as we later point out, equality may not always be the case and, in certain situations, behavior following one stimulus may be more (or less) sensitive to reinforcement variation than that emitted following a different stimulus.

Isobias and alloiobias. Parametric variation of reinforcement schedules has received little consideration in signal-detection research. For an adequate isosensitivity contour, an independent variable which controls or biases behavior is clearly needed, and, as noted above, these variables have been well specified by concurrent-schedule research. The list includes reinforcement rate, reinforcement magnitude, immediacy of reinforcement, quality of reinforcement (de Villiers, 1977), and response requirements (Beautrais \& Davison, 1977; Davison \& Ferguson, 1978). Recent research in this laboratory on response force suggests that, because of its extremely fast effect on behavior, this biaser may be particularly efficient.

The present model views response bias as a function of the obtained reinforcement ratio rather than of the arranged payoff matrices. Thus, it can specify true iso- (equal-) bias contours, and these will be generated only in the case in which the ratio of reinforcements obtained for correct responses is kept constant. Isobias contours predicted by this model for such a controlled reinforcement-ratio procedure are shown in Figure $5 \mathrm{a}$ for various degrees of bias. Response bias is given by Equation 11, and comprises inherent bias $(\log c)$ and bias due to the reinforcement ratio. Thus, a response bias of .3 may represent any combination of inherent and reinforcement bias. The shape of the predicted isobias contour is the same as Dusoir (1975) drew for Luce's (1963) model. However, Luce's model was written for the case in which the obtained reinforcement ratio covaried with the response ratio, that is, the standard signal-detection procedure.

Our model makes very different predictions for such an uncontrolled reinforcement-ratio procedure. When reinforcement ratios are not controlled, and stimulus-presentation probability is held constant, changes in behavior will change reinforcement ratios obtained, which will again change behavior, and so on. Some idea of the expected alloiobias (varying, different bias) contour can be obtained by applying Equations 3 and 4 a number of times. The results are shown in Figure 5b. Here, we have assumed that stimulus-presentation probability is .5 , the initial reinforcement ratio is 1.0 , and $a_{r}$ is 1.0 , and that the subject has an inherent bias of .25 to response $P_{1}$ (Figure 2). Line 1 is a controlled equal-reinforcement

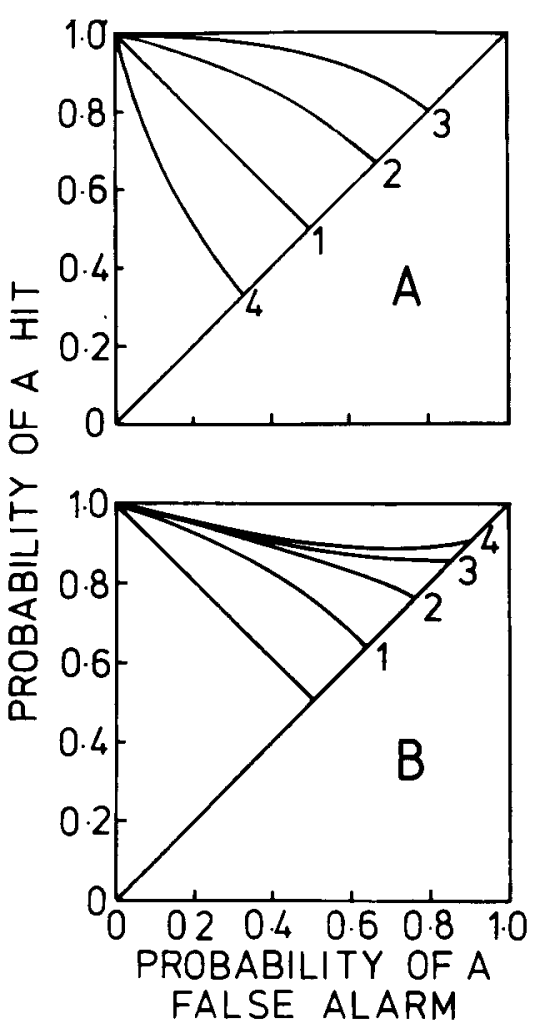

Figure 5. Predicted isobias contours under various assumptions. In Graph $A$, the obtained reinforcement ratio is controlled (see text). The value of $\left[\log c+\log \left(R_{W} / R_{Z}\right)\right]$ is 0 (line 1$), .3$ (line 2), .6 (line 3 ), and -.3 (line 4$)$. In Graph $B$, the obtained reinforcement ratio is not controlled, and is a function of changing preference. Line 1 shows a predicted isobias contour when inherent bias is .25 , and lines 2,3 , and 4 show the further development of the contour as changing preference affects the obtained reinforcements for the two choices.

procedure with the above parameters. From here on, the reinforcement ratio is uncontrolled, and it is the inherent bias of .25 , and consequent inequality of obtained reinforcements for the two choices, that moves the contour toward the upper-right corner of the ROC space. Line 2 is predicted by the first application of Equations 3 and 4, Line 3 by the second, and Line 4 by the third. Thus, as training progresses with a particular pair of stimuli, the data point will move progressively toward the upper-right corner of the ROC space. The complete alloiobias contour, obtained from a number of stimulus pairs, will take the shape shown in Figure 5b, that is, the shape usually reported in the literature, and the shape predicted by a signal-detection theory maximizing expected value approach (Dusoir, 1975). (Likewise, if inherent bias was -.25 , successive applications of Equations 3 and 4 would move the contour in Figure 5b progressively toward the lower-left corner of the ROC space.)

Preference is clearly moving faster with the poor discriminability stimuli (near the major diagonal) 
than with good discriminability. In theory, the process should stop with exclusive choice for response $P_{1}$, but if the subject is insensitive to small reinforcementratio changes, the curve will stabilize with a strongly negative decelerated shape. It is, of course, possible that adventitious changes in reinforcement ratios obtained may take the alloiobias line across the minor diagonal. The point we wish to make, however, is that the alloiobias function is predictable from a consideration of the obtained reinforcement ratios, whereas it is not predictable from stimulus-presentation probabilities. In addition, the problems with uncontrolled bias shifts in detection-theory research (e.g., Hobson, 1975; Terman \& Terman, 1972) can now be seen as problems in defining biasing variables in relation to experimental procedures.

\section{Implications for Human Psychophysics}

The present approach to bias in signal detection has concentrated almost entirely on animal studies. To what extent do the results discussed apply to human psychophysics? The answers to this question are problematical, and seem to depend rather more on one's theoretical stance than on data. The traditions of behavioral and psychophysical research are different, and they have never been adequately combined. Most research in animal psychophysics has simply been carried out to extend or generalize principles from human research. We, of course, are taking an opposing view: that animal research can contribute generally to psychophysics and can highlight theoretical difficulties which arise purely from using human subjects.

From our radical behaviorist viewpoint, it is necessary (except when using respondents) to maintain the behavior of the animal explicitly through reinforcement. Motivational or, more strictly, reinforcement variables must thus be clearly specified and be arranged adequately to maintain detection performance. In the absence of reinforcement, detection performance ceases. More than this, differential reinforcement is required as shown by Davison and McCarthy (1980). From our own research, and by implication from published research on concurrent schedules, we know many things about reinforcement effects: that the degree of deprivation should not affect either response bias or discriminability (except when different reinforcers are used for the two correct responses); that overall reinforcement rate does not affect either response bias or discriminability; that the presence or absence of punishment for errors, in the context of reinforcement for correct responses, does not change response bias or discriminability; that reinforcement for errors decreases discriminability; that response bias can be predictably changed by a wide range of variables.

On the other hand, in our research we have yet to see any unambiguous effects of expectancy on re- sponse bias. As we have shown, response bias in animal research is unaffected by SPP variation alone, and extreme SPP values do not produce response biases when equal payoff is given for the two correct responses. Is it more parsimonious to assume, then, that humans and animals are different in the effect that presentation frequency has on response bias? Because the relevant experiment has not been carried out with humans, we assume that the same principles apply, and that, in the human case, insufficient attention has been given to specifying the reinforcers involved.

In human experimentation, the reinforcers (money, grades, etc.) are often delivered non-contingently, as a means simply of providing and maintaining behavior. The differential reinforcers reside in the instructions, and these do have a significant bearing on the strategies adopted. Such instructions as "Report the presence of the tone only when you are absolutely sure it is present" obliquely specify that the frequency, or probability, of payoff for the response "absent" is greater than that for the response "present." It is thus a biasing operation, as is explicit reinforcement variation with animals. Variations of SPP in conjunction with an instruction such as "Say yes when the tone is present and no when it is absent" are an oblique variation of reinforcement frequency. But what of the instruction, "You will occasionally receive 10 cents each time you report the tone when it is present, and when you report it absent and it is indeed absent. You have 100 trials in which to make as much money as you can"? If the frequencies of reinforcement for "yes" and "no" were kept constant while SPP was varied, would response bias change? Likewise, if SPP was held constant and reinforcement frequency for the two reports was varied, would response bias change?

We thus argue that animal detection-theory research is, in some sense, more informative than human research because it forces us better to specify the variables involved. Only human research can tell us if this is the correct approach.

At the data level, the commonalities between the results of animal and human experimentation are enormous, as we have shown. The problems that signal-detection theory has had with "bias" (or criterion), and the form of the isobias contour (Dusoir, 1975), to take one instance, has been due to a very large extent to the inadequate specification of the variables that lead to bias, as Dusoir pointed out. Another particular example can be cited. The rather oft-occurring nonunit slope of the isosensitivity function on double $\mathrm{z}$ coordinates has been ascribed to criterion variance (e.g., Wickelgren, 1968). From our theoretical standpoint, because we see response bias as determined largely by environmental (reinforcement) contingencies, we must expect the variance in response bias as also being so determined. It is an em- 
pirical question, then, whether trial-to-trial or session to-session variance in obtained reinforcement ratios can decrease the slope of the isosensitivity function. From our viewpoint, then, criterion variance does not simply exist-it is manipulable and investigatable. Equally, from our theoretical standpoint, a nonunit slope implies that behavior following one stimulus is more sensitive to reinforcement variation than is that emitted following another (i.e., $a_{r_{1}} \neq a_{r_{2}}$ in Equations 3 and 4). Indeed, we have found this in some experiments as yet unpublished, and we are presently investigating the conditions under which this inequality occurs.

In general, then, our approach to bias and its operation could have some wide applications in psychophysics. It does not attempt to supplant conventional detection theory; rather, it attempts to provide it with an adequate theory of response bias derived from an area in which such effects have been very extensively researched. As we have stated elsewhere, the study of stimulus and reinforcement effects should not proceed separately when each approach requires a consideration of the other. Thus, in addition to clarifying the role of reinforcement in psychophysics, our work is also addressed to the adequate measurement of stimulus effects in the situations conventionally studied under the heading of choice and schedule control. Such cross-fertilization between two highly developed areas of psychology will surely lead to benefits for both.

\section{REFERENCE NOTE}

1. Nevin, J. A., Jenkins, P., Whittaker, S., \& Yarensky, P. Signal detection, differential reinforcement and matching. Paper presented at the meeting of the Psychonomic Society, Washington, D.C., November 1977.

\section{REFERENCES}

Barron, B., \& Davison, M. Performance in multiple fixedinterval schedules. Journal of the Experimental Analysis of Behavior, 1972, 17, 375-379.

BAUM, W. M. On two types of deviation from the matching law: Bias and undermatching. Journal of the Experimental Analysis of Behavior, 1974, 22, 231-242.

BAUM, W. M. Matching, undermatching, and overmatching in studies of choice. Journal of the Experimental Analysis of Behavior, 1979, 32, 269-281.

BaUM, W. M., \& RACHLIN, H. C. Choice as time allocation. Journal of the Experimental Analysis of Behavior, 1969, 12, 861-874.

Beautrais, P. G., \& Davison, M. C. Response and time allocation in concurrent second-order schedules. Journal of the Experimental Analysis of Behavior, 1977, 25, 61-69.

Broadbent, D. E. Decision and stress. London: Academic Press, 1971.

Clopton, B. M. Detection of increments in noise intensity by monkeys. Journal of the Experimental Analysis of Behavior, $1972,17,473-481$.

Davison, M. C. A functional analysis of chained fixed-interval schedule performance. Journal of the Experimental Analysis of Behavior, 1974, 21, 323-330.
Davison, M. C., \& Ferguson, A. The effects of different component response requirements in multiple and concurrent schedules. Journal of the Experimental Analysis of Behavior, 1978, 29, 283-295.

Davison, M., \& McCArthy, D. Reinforcement for errots in a signal-detection procedure. Journal of the Experimental Analysis of Behavior, 1980, 34, 35-47.

Davison, M. C., \& Tustin, R. D. The relation between the generalized matching law and signal-detection theory. Journal of the Experimental Analysis of Behavior, 1978, 29, 331-336.

DE VILLIERS, P. Choice in concurrent schedules and a quantitative formulation of the law of effect. In W. K. Honig \& J. E. R. Staddon (Eds.), Handbook of operant behavior. Englewood Cliffs, N.J: Prentice-Hall, 1977.

Dusoir, A. E. Treatments of bias in detection and recognition models: A review. Perception \& Psychophysics, 1975, 17, 167-178.

EgAN, J. P. Signal detection theory and ROC analysis. New York: Academic Press, 1975.

ELSMORE, T. F. Duration discrimination: Effects of probability of stimulus presentation. Journal of the Experimental Analysis of Behavior, 1972, 18, 465-469.

Galanter, E., \& Holman, G. L. Some invariances of the isosensitivity functions and their implications for the utility function of money. Journal of Experimental Psychology, 1967, 73 , 333-339.

Green, D. M., \& Swets, J. A. Signal detection theory and psychophysics. New York: Wiley, 1966.

HARDY, G. R., \& LEgGe, D. Cross-modal induction of changes in sensory thresholds. Quarterly Journal of Experimental Psy. chology, 1968, 20, 20-29.

Healy, A. F., \& Jones, C. Criterion shifts in recall. Psychological Bulletin, 1973, 79, 335-340.

Herrnstein, R. J. On the law of effect. Journal of the Experimental Analysis of Behavior, 1970, 13, 243-266.

Herrnstein, R. J., \& Heyman, G. M. Is matching compatible with reinforcement maximization on concurrent variable interval, variable ratio? Journal of the Experimental Analysis of Behavior, 1979, 31, 209-223.

HoBson, S. L. Discriminability of fixed-ratio schedules for pigeons: Effects of absolute ratio size. Journal of the Experimental Analysis of Behavior, 1975, 23, 25-35.

Hollard, V. D., \& Davison, M. C. Preference for qualitatively different reinforcers. Journal of the Experimental Analysis of Behavior, 1971, 16, 375-380.

Hume, A. L. Auditory detection and optimal response biases. Perception \& Psychophysics, 1974, 15, 425-433. (a)

Hume, A. L. Optimal response biases and the slope of ROC curves as a function of signal intensity, signal probability, and relative payoff. Perception \& Psychophysics, 1974, 16, 377-384. (b)

Hume, A. L., \& Inwin, R. J. Bias functions and operating characteristics of rats discriminating auditory stimuli. Journal of the Experimental Analysis of Behavior, 1974, 21, 285-295.

IRWIN, R. J., \& Terman, M. Detection of brief tones in noise by rats. Journal of the Experimental Analysis of Behavior, 1970, $13,135-143$.

LANDER, D. G., \& Inwin, R. J. Multiple schedules: Effects of the distribution of reinforcements between components on the distribution of responses between components. Journal of the Experimental Analysis of Behavior, 1968, 11, 517-524.

LoBB, B., \& DAvison, M. C. Preference in concurrent interval schedules: A systematic replication. Journal of the Experimental Analysis of Behavior, 1975, 24, 191-197.

Luce, R. D. Individual choice behavior. New York: Wiley, 1959. Luce, R. D. Detection and recognition. In R. D. Luce, R. R. Bush, \& E. Galanter (Eds.), Handbook of mathematical psychology (Vol. 1). New York: Wiley, 1963.

Markowitz, J., \& Swets, J. A. Factors affecting the slope of empirical ROC curves: Comparison of binary and rating responses. Perception \& Psychophysics, 1967, 2, 91-100. 
Mathews, L. R., \& Temple, W. Concurrent schedule assessment of food preference in cows. Journal of the Experimental Analysis of Behavior, 1979, 32, 245-254.

McCarthy, D., \& Davison, M. Signal probability, reinforcement, and signal detection. Journal of the Experimental Analysis of Behavior, 1979, 32, 373-386.

McCanthy, D., \& Davison, M. On the discriminability of stimulus duration. Journal of the Experimental Analysis of Behavior, 1980, 33, 187-211. (a)

McCarthy, D., \& Davison, M. Independence of sensitivity to relative reinforcement rate and discriminability in signal detection. Journal of the Experimental Analysis of Behavior, 1980, 34, 273-284. (b)

MYERs, D. L., \& MYERs, L. E. Undermatching: A reappraisal of performance on concurrent variable-interval schedules of reinforcement. Journal of the Experimental Analysis of Behavior, 1977, 27, 203-214.

Nevin, J. A. Rates and patterns of responding with concurrent fixed-interval and variable-interval reinforcement. Journal of the Experimental Analysis of Behavior, 1971, 16, 241-247.

Parks, T. E. Signal detectability theory of recognition memory performance. Psychological Review, 1966, 73, 44-58.

Peterson, W. W., Birdsall, T. G., \& Fox, W. C. The theory of signal detectability. Transactions of the IRE Professional Group on Information Theory, PGIT-4, 1954.

SchNe ide R, J. W. Reinforcer effectiveness as a function of reinforcer rate and magnitude: A comparison of concurrent performances. Journal of the Experimental Analysis of Behavior, $1973,20,461-471$.

Schulman, A. I., \& Greenberg, G. Z. Operating characteristics and a priori probability of the signal. Perception \& Psychophysics, 1970, 8, 317-320.

Staddon, J. E. R. Spaced responding and choice: A preliminary analysis. Journal of the Experimental Analysis of Behavior, $1968,11,669-682$.

StubBs, D. A. Response bias and the discrimination of stimulus duration. Journal of the Experimental Analysis of Behavior, $1976,25,243-250$.

Swets, J. A., TAnner, W. P., Jr., \& Birdsall, T. G. Decision processes in perception. Psychological Review, 1961, 68, 301-340.
TAnner, W. P., Jr., \& Swets, J. A. A decision-making theory of visual detection. Psychological Review, 1954, 61, 401-409.

Terman, M., \& Terman, J. S. Concurrent variation of response bias and sensitivity in an operant-psychophysical test. Perception \& Psychophysics, 1972, 11, 429-432.

Thomas, E. A. C., \& Legge, D. Probability matching as a basis for detection and recognition decisions. Psychological Review, $1970,77,65-72$.

ToDonov, J. C. Interaction of frequency and magnitude of reinforcement on concurrent performances. Journal of the Experimental Analysis of Behavior, 1973, 19, 451-458.

Treisman, M. The effect of one stimulus on the threshold for another: An application of signal detectability theory. British Journal of Statistical Psychology, 1964, 17, 15-35.

Treisman, $M$. On the use and misuse of psychophysical terms. Psychological Review, 1976, 83, 246-256.

Treisman, M. On the stability of $\mathrm{d}_{\mathrm{s}}$. Psychological Bulletin, 1977. 84, 235-243.

Trevett, A. J., Davison, M. C., \& Williams, R. J. Performance in concurrent interval schedules. Journal of the Experimental Analysis of Behavior, 1972, 17, 369-374.

van Meter, D., \& Middleton, D. Modern statistical approaches to reception in communication theory. Transactions of the IRE Professional Group on Information Theory, PGIT 4 , 1954.

WickelgRen, W. A. Unidimensional strength theory and component analysis of noise in absolute and comparative judgments. Journal of Mathematical Psychology, 1968, 5, 102-122.

\section{NOTE}

1. Nevin, Jenkins, Whittaker, and Yarensky (Note 1) proposed a model similar to that reported here. Their model, which assumes $a_{T}=1$ and $c=1$, is thus a special case of our model. The relative merits of these two models, and the necessity for the generalized model, are discussed in Davison and McCarthy (1980).

(Received for publication December 7, 1979; revision accepted February 9,1981 .) 